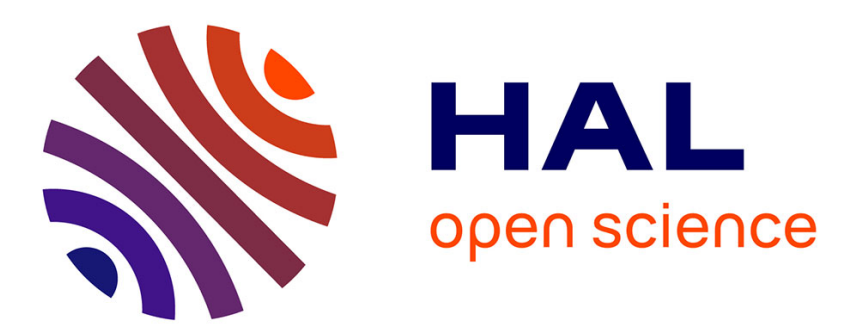

\title{
Une méthode semi-explicite pour la simulation des problèmes d'impact avec frottement
}

Jean-Michel Cros, Zhi-Qiang Feng, Benoit Magnain

\section{To cite this version:}

Jean-Michel Cros, Zhi-Qiang Feng, Benoit Magnain. Une méthode semi-explicite pour la simulation des problèmes d'impact avec frottement. Comptes Rendus Mécanique, 2010, 338 (3), pp.176-180. 10.1016/j.crme.2010.03.007 . hal-01176165

\section{HAL Id: hal-01176165 \\ https://hal.science/hal-01176165}

Submitted on 3 Jul 2018

HAL is a multi-disciplinary open access archive for the deposit and dissemination of scientific research documents, whether they are published or not. The documents may come from teaching and research institutions in France or abroad, or from public or private research centers.
L'archive ouverte pluridisciplinaire HAL, est destinée au dépôt et à la diffusion de documents scientifiques de niveau recherche, publiés ou non, émanant des établissements d'enseignement et de recherche français ou étrangers, des laboratoires publics ou privés. 


\title{
Une méthode semi-explicite pour la simulation des problèmes d'impact avec frottement
}

\section{A semi-explicit method to simulate frictional impact problems}

\author{
Jean-Michel Cros ${ }^{\mathrm{a}, *}$, Zhi-Qiang Feng ${ }^{\mathrm{a}}$, Benoît Magnain ${ }^{\mathrm{b}}$ \\ ${ }^{a}$ Laboratoire LME-Evry, Université d'Évry - Val d'Essonne, 91020 Évry, France \\ ${ }^{\mathrm{b}}$ Institut PRISME, ENSI de Bourges, 88 bd. Lahitolle, 18020 Bourges, France
}

\begin{abstract}
Résumé On s'intéresse à la modélisation des problèmes d'impact avec frottement. Cette Note présente une approche qui concilie la rapidité de résolution (calcul semi-explicite) et la finesse de modélisation des zones de contact par la méthode du bi-potentiel. Cette dernière méthode traite le contact unilatéral et le frottement de manière couplée via un bi-potentiel de contact ce qui conduit à une résolution implicite limitée aux zones des contacts entre les corps déformables avec prise en compte ou non du frottement. La méthode proposée est comparée à une approche implicite implantée dans le code FER/Impact et à une méthode explicite disponible dans le code de calcul industriel LS-DYNA.
\end{abstract}

\begin{abstract}
We are interested in the modeling of frictional impact problems. This Note presents an approach which reconciles the speed of resolution (semi-explicit calculation) and the accuracy of contact modeling by the bi-potential method. This method deals with the unilateral contact and the friction in a coupling way via a contact bi-potential. It leads to an implicit resolution at the contact zones between deformable bodies with or without friction. The proposed method is compared with an implicit one both implemented in the code FER/Impact and with a pure explicit method available in the industrial code LS-DYNA.
\end{abstract}

\section{Abridged English version}

In this study, we consider frictional impact problems with large displacements. The total Lagrangian formulation is used for the finite element discretization. The equation of motion (Eq. (1)) is integrated by means of an explicit time stepping scheme (Eq. (2)) which leads to a direct resolution without construction and factorization of global matrices. However, the contact forces are solved in an implicit manner by the bi-potential method [1,2]. It is important to note that, as opposed to the penalty method or Lagrange multiplier method, the bi-potential method neither changes the global stiffness matrix, nor increases the degrees of freedom. In addition, the solution procedure is more stable because of the separation of nonlinearities and improved numerical algorithms for calculation of contact forces.

\footnotetext{
* Auteur correspondant.

Adresse e-mail : cros@iup.univ-evry.fr (J.-M. Cros).
} 
The proposed semi-explicit method is compared with a previously developed implicit method available in FER/Impact [3] and with a pure explicit method available in the industrial code LS-DYNA [4,5]. To illustrate the effectiveness and the robustness of the algorithm, we consider a test example which concerns the oblique impact of an elastic plate onto a rigid surface with rebounding (Fig. 1). We observe from Fig. 2 that the distribution of von Mises stress is in good concordance between the semi-explicit and implicit schemes. In both cases, the localization of the maximum stress is inside the plate rather than at the contact surface. Table 1 shows the CPU time for different configurations (scheme, mesh, time step). It is clearly shown that the semi-explicit scheme is much more restrictive with respect to the stability. However, if the time step is small enough, the semi-explicit scheme is much more efficient (as expected) than the implicit one in terms of CPU time. Fig. 3 shows the plots of the kinetic energy $\left(E_{c}\right)$, the elastic strain energy $\left(E_{e}\right)$ and the total energy $\left(E_{t}=E_{c}+E_{e}\right)$ obtained respectively by FER/Impact and LS-DYNA. These results show better property of energy conservation of the proposed semiexplicit method over the pure explicit one. It is also interesting to analyze the results by focalizing on the accuracy of the semi-explicit solution as compared with the implicit one. Fig. 4 gives relative errors of the total energy made by the two schemes at different configurations. Globally, we observe that the implicit scheme allows to preserve better the total energy than the semi-explicit scheme. However, the behavior of the semi-explicit scheme is acceptable because the error is relatively small (in general, less than $2 \%$ as shown in Fig. 4).

\section{Introduction}

Dans cette Note, on s'intéresse aux problèmes d'impact et de choc. Il s'avère que pour certaines simulations en dynamique rapide, il convient d'obtenir une information précise sur les champs de pression au niveau des zones de contact. Or, par nature les méthodes de pénalisation que l'on trouve dans les codes industriels donnent une information imprécise du fait de la gestion incorrecte de la pénétration au niveau des zones de contact [6]. La méthode du bi-potentiel a fait largement ses preuves pour la simulation des problèmes de contact avec ou sans frottement en statique et dynamique (implicite) [1,3]. Ces méthodes sont implantées dans le code FER/Impact [3]. On se propose de coupler cette méthode à un schéma d'intégration explicite afin d'essayer de concilier la rapidité de résolution et la finesse de modélisation des zones de contact.

\section{Modélisation et discrétisation}

On s'intéresse aux problèmes d'impact avec frottement dans le cadre des grands déplacements. Le comportement des matériaux peut être élastique et/ou hyperélastique. La discrétisation utilise la formulation lagrangienne totale et la méthode des éléments finis. Le problème de dynamique non-linéaire avec prise en compte du contact s'écrit comme suit :

$$
\mathbf{M} \ddot{\mathbf{U}}+\mathbf{A} \dot{\mathbf{U}}+\mathbf{F}_{\text {int }}(\mathbf{U})-\mathbf{F}_{\text {ext }}-\mathbf{R}=0
$$

Les vecteurs $\mathbf{F}_{\text {int }}(\mathbf{U}), \mathbf{F}_{\text {ext }}$ et $\mathbf{R}$ représentent respectivement les forces internes qui sont fonction du vecteur des déplacements $\mathbf{U}$, les forces extérieures et les réactions de contact. $\mathbf{M}$ est la matrice de masse et $\mathbf{A}$ est la matrice d'amortissement. Les vecteurs $\dot{\mathbf{U}}$ et $\ddot{\mathbf{U}}$ correspondent respectivement aux vitesses et aux accélérations. L'équation (1) est fortement non-linéaire, notamment en raison des grandes rotations et des grands déplacements des corps que l'on rencontre dans les problèmes d'impact multicorps. De plus, la loi qui gouverne le contact avec frottement est représentée par des inégalités et le potentiel de contact est non différentiable. Ce problème a été déjà traité par une approche implicite qui utilise un schéma d'intégration du premier ordre [3]. Dans cet article, on utilise un schéma d'intégration explicite basé sur les approximations suivantes (différences finies centrées) :

$$
\dot{\mathbf{U}}_{t}=\frac{1}{2 \Delta t}\left(\mathbf{U}_{t+\Delta t}-\mathbf{U}_{t-\Delta t}\right), \quad \ddot{\mathbf{U}}_{t}=\frac{1}{\Delta t^{2}}\left(\mathbf{U}_{t+\Delta t}-2 \mathbf{U}_{t}+\mathbf{U}_{t-\Delta t}\right)
$$

En considérant, en plus, le cas sans amortissement, on obtient :

$$
\mathbf{U}_{t+\Delta t}=\Delta t^{2} \mathbf{M}^{-1}\left(\left(\mathbf{F}_{\text {ext }}\right)_{t}-\left(\mathbf{F}_{\text {int }}\right)_{t}+\mathbf{R}_{t+\Delta t}\right)+2 \mathbf{U}_{t}-\mathbf{U}_{t-\Delta t}
$$

Plutôt que d'essayer de tenir compte en même temps de toutes les non-linéarités du problème, une stratégie consiste à les séparer. Pour ce faire l'équation (3) est écrite sous la forme suivante :

$$
\mathbf{U}_{t+\Delta t}=\left(\mathbf{U}_{l i b}\right)_{t}+\left(\mathbf{U}_{c}\right)_{t+\Delta t}
$$

Le terme $\mathbf{U}_{\text {lib }}$ correspond à la solution du problème sans tenir compte du contact et le terme $\mathbf{U}_{c}=\Delta t^{2} \mathbf{M}^{-1} \mathbf{R}$ représente les déplacements engendrés par les forces de contact. Dans un repère local (vecteur normal $\mathbf{n}$ et tangentiel $\mathbf{t}$ ), les réactions de chaque nœud de contact (r) sont calculées par une procédure itérative de prédiction-correction :

$$
\begin{aligned}
& \text { Prédiction : } \mathbf{r}^{*(i+1)}=\mathbf{r}^{(i)}-\rho^{(i)}\left(\mathbf{x}^{(i)}+\mu\left\|\mathbf{x}_{t}^{(i)}\right\| \mathbf{n}\right) \\
& \text { Correction }: \mathbf{r}^{(i+1)}=\operatorname{Proj}_{\mathbf{K}_{\mu}}\left(\mathbf{r}^{*(i+1)}\right)
\end{aligned}
$$

où $\mathbf{K}_{\mu}$ représente le cône de Coulomb et $\mathbf{x}$ représente la position relative au point de contact. En raison de cette approche l'algorithme n'est pas purement explicite. En effet, les forces extérieures sont traitées de manière explicite mais ce n'est pas 

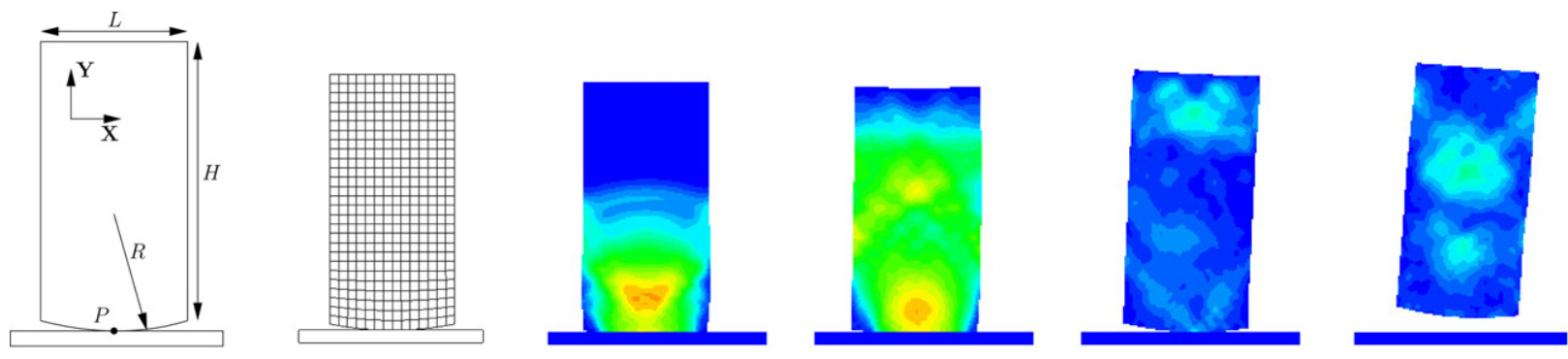

Fig. 1. Impact oblique d'une plaque élastique : géométrie, maillage (M1) et déformations successives $(\mu=0,1)$.

Fig. 1. Oblique impact of an elastic plate: geometry and mesh (M1), evolution of von Mises stress $(\mu=0.1)$.

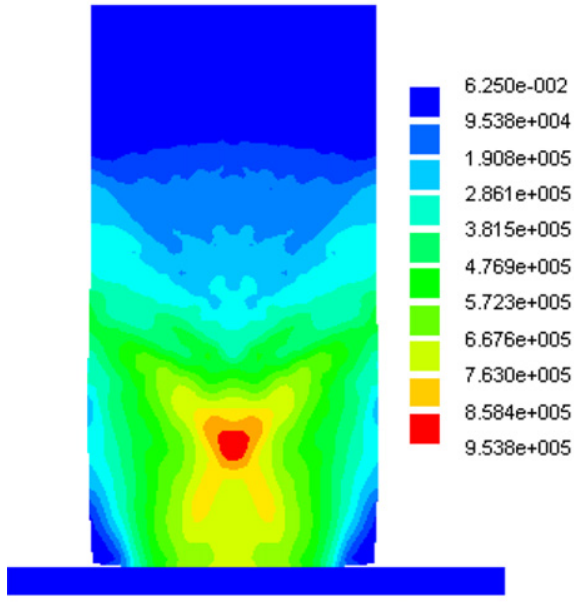

Méthode implicite

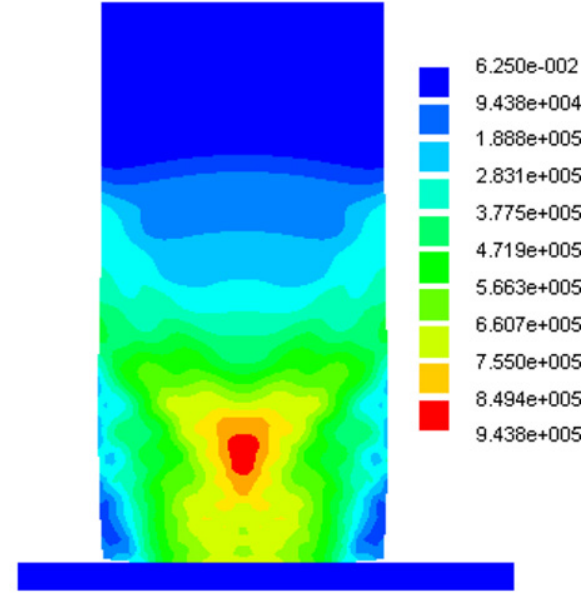

Méthode semi-explicite

Fig. 2. Distribution des contraintes équivalentes de von Mises $(\mu=0,0)$ à $t=0,0006 \mathrm{~s}$.

Fig. 2. Distribution of von Mises stress $(\mu=0.0)$ at $t=0.0006 \mathrm{~s}$.

le cas des forces de contact qui sont obtenues de manière implicite par la méthode de bi-potentiel (résolution d'un problème condensé sur les nœuds de contact) [2]. En conséquence, la méthode proposée est qualifiée de semi-explicite. Ce schéma est conditionnellement stable. Le pas de temps $\Delta t$ doit être inférieur à une valeur critique qui est liée notamment à la taille des éléments et à la vitesse de propagation des ondes dans le corps que l'on peut estimer $\Delta t_{c}=\frac{\ell}{\sqrt{E / \rho}}$. Cette condition peut s'avérer pénalisante pour les problèmes d'impact car la zone de contact est généralement finement maillée. Si la matrice de masse est (ou est rendue) diagonale, le système d'équations peut se résoudre sans factorisation de la matrice de masse effective. En conséquence, le coût d'une itération est très faible par rapport à celui d'une méthode implicite.

\section{Résultats numériques}

On traite un problème classique d'impact [7] de type Neumann homogène (Fig. 1). Les caractéristiques mécaniques sont les suivantes : module de Young $E=10^{7} \mathrm{~Pa}$, coefficient de Poisson $v=0,25$, masse volumique $\rho=1000 \mathrm{~kg} / \mathrm{m}^{3}$, vitesse initiale : $\dot{U}_{x}=3 \mathrm{~m} / \mathrm{s}, \dot{U}_{y}=-5 \mathrm{~m} / \mathrm{s}$. Les dimensions sont données par : $L=0,04 \mathrm{~m}, H=0,08 \mathrm{~m}$, rayon $R=0,101 \mathrm{~m}$, épaisseur $e=0,01 \mathrm{~m}$. Le temps de simulation est de $3 \times 10^{-3} \mathrm{~s}$ et différents pas de temps compris entre $10^{-4} \mathrm{~s}$ et $10^{-6} \mathrm{~s}$, sont utilisés pour étudier la stabilité de la méthode. Les résultats sont comparés à ceux obtenus par une méthode implicite [3] et par une méthode explicite présente dans un code industriel (LS-DYNA).

Deux maillages sont utilisés afin d'évaluer la sensibilité au pas de discrétisation spatiale. Le premier, noté M1 (Fig. 1), comprend 379 éléments et 424 nœuds alors que le second, noté M2, comporte 3281 éléments et 3409 nœuds.

Dans un premier temps, le frottement n'est pas pris en compte $(\mu=0,0)$. Au cours de la simulation, la contrainte maximale de von Mises atteinte est de $9,438 \times 10^{5} \mathrm{~Pa}$, cette valeur est légèrement inférieure au résultat obtenu par un schéma d'intégration implicite $\left(\sigma_{\max }=9,538 \times 10^{5} \mathrm{~Pa}\right)$. On note, Fig. 2 , une bonne concordance de la distribution des contraintes de von Mises entre les schémas semi-explicite et implicite. Dans les deux cas, la contrainte maximale se situe à l'intérieur de la plaque et non au niveau de la surface de contact. 
Tableau 1

Comparaison des temps de calculs.

Table 1

Comparison of CPU time.

\begin{tabular}{lllllc}
\hline Maillage & M1 & M1 & M1 & M2 & M2 \\
\hline$\Delta t(\mathrm{~s})$ & $10^{-4}$ & $10^{-5}$ & $10^{-6}$ & $10^{-4}$ & $10^{-5}$ \\
Semi-explicite (s) & divergence & 5,68 & 14,63 & divergence & divergence \\
Implicite (s) & 8,44 & 75,65 & 446,56 & 484,01 & 3907,23 \\
\hline
\end{tabular}
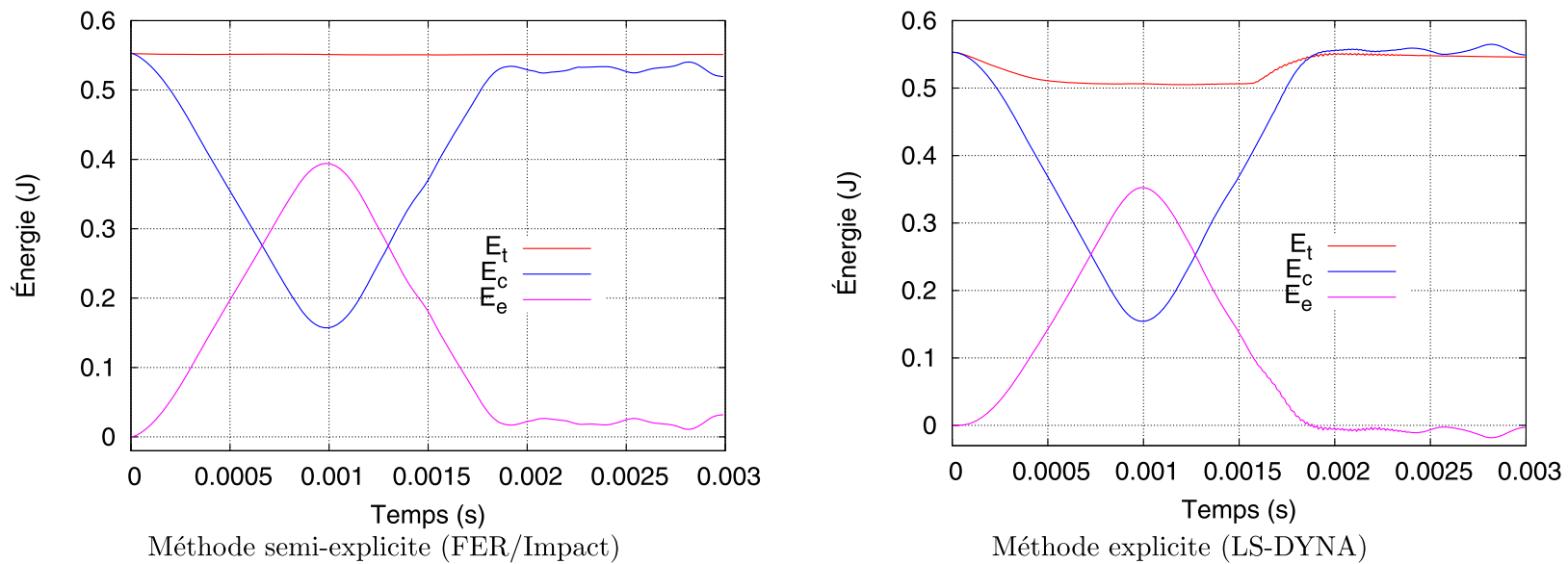

Fig. 3. Évolution des énergies, maillage M2.

Fig. 3. Energy variation, mesh M2.

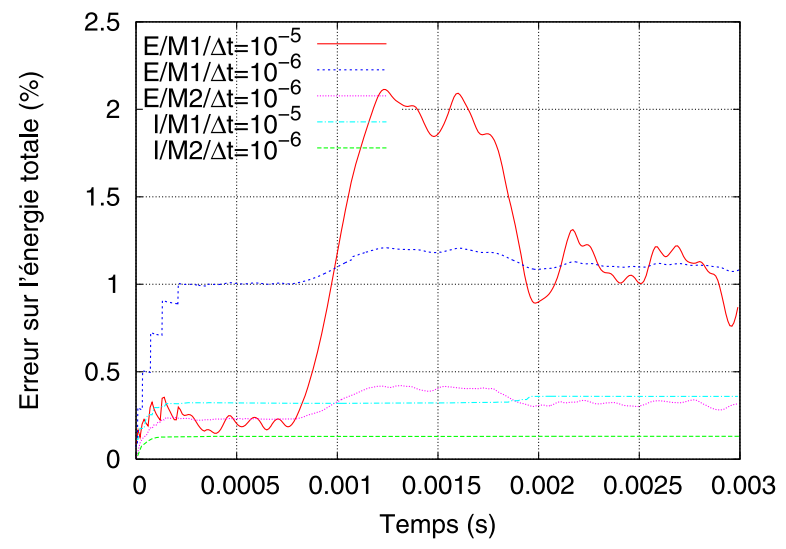

Fig. 4. Erreur sur l'énergie totale.

Fig. 4. Total energy error.

Le Tableau 1 récapitule les temps de calcul pour différentes configurations (schéma, maillage, pas de temps). Le caractère conditionnel du schéma semi-explicite apparaît clairement, toutefois même avec un pas de temps très petit afin de garantir la stabilité du schéma, les temps de calcul sont très nettement inférieurs à ceux de l'approche implicite.

Pour analyser la précision de la solution, on s'intéresse à la conservation de l'énergie. La Fig. 3 montre l'évolution de l'énergie cinétique $\left(E_{c}\right)$, de l'énergie de déformation élastique $\left(E_{e}\right)$ et de la somme $\left(E_{t}=E_{c}+E_{e}\right)$. Le résultat obtenu par l'approche semi-explicite est bien meilleur que celui obtenu par l'approche purement explicite (LS-DYNA, pas de temps moyen $8,25 \times 10^{-6} \mathrm{~s}$ ).

La Fig. 4 montre l'évolution de l'erreur sur $E_{t}$ pour différentes configurations. On note que le schéma implicite représente mieux l'énergie totale toutefois le résultat obtenu par le schéma semi-explicite est acceptable car l'erreur est le plus souvent inférieure à $2 \%$.

Les Figs. 5 et 6 montrent l'évolution des déplacements horizontal $U_{x}$ et vertical $U_{y}$ du point $P$ déterminé par un calcul implicite et semi-explicite. Il faut souligner que la condition d'impénétrabilité est parfaitement satisfaite par notre approche, ce qui n'est pas le cas avec LS-DYNA. 


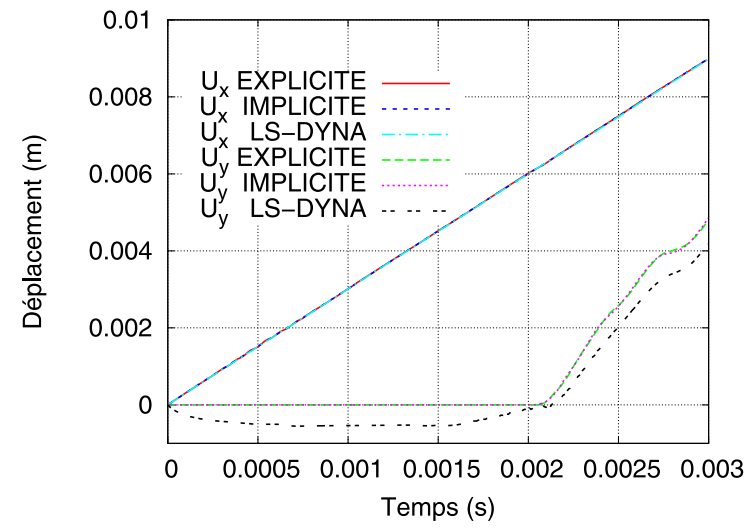

Fig. 5. Déplacement du point $P$ sans frottement.

Fig. 5. Displacement of point $P$ without friction.

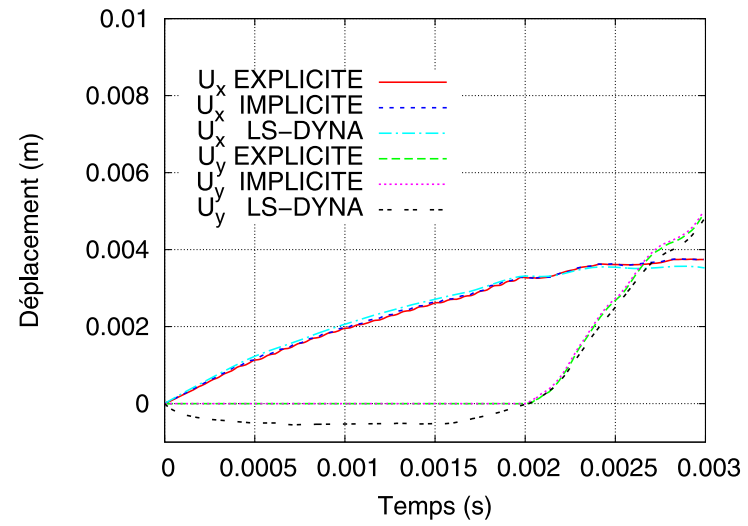

Fig. 6. Déplacement du point $P$ avec frottement $(\mu=0,1)$.

Fig. 6. Displacement of point $P$ with friction $(\mu=0.1)$.

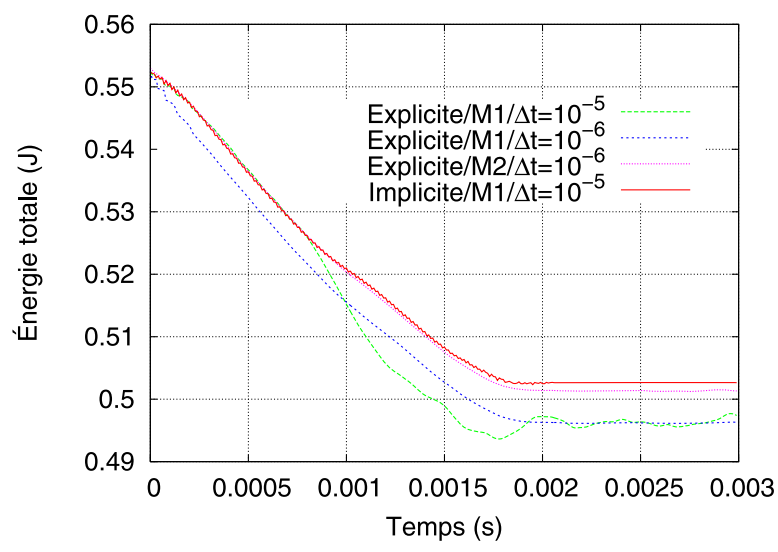

Fig. 7. Dissipation de l'énergie totale.

Fig. 7. Total energy dissipation.

On considère à présent le cas avec frottement $(\mu=0,1)$. La Fig. 1 représente les déformées sur lesquelles sont tracées les contraintes de von Mises à différents instants : 0,0005 s;0,001 s; 0,002 s et 0,003 s. La Fig. 7 représente l'évolution de l'énergie totale qui décroît en raison de la présence du frottement. On remarque que le choix d'un maillage approprié et d'un pas de temps adapté permet d'améliorer la simulation et de tendre vers la solution implicite.

\section{Conclusions}

Les résultats et la comparaison avec une méthode implicite montrent que l'approche proposée intègre une bonne modélisation des réactions de contact grâce à la méthode du bi-potentiel et s'avère extrêmement rapide d'un point de vue du temps de calcul. Par ailleurs, la méthode proposée gère de manière beaucoup plus correcte et sans artifice l'énergie comparativement à une méthode purement explicite. La méthode a également été testée avec succès sur des problèmes tridimensionnels avec des lois de comportement hyperélastiques de type Mooney-Rivlin. Le code doit être optimisé et d'autres fonctions doivent être ajoutées comme la gestion de l'effet sablier (Hourglass) ou la possibilité d'avoir un pas de temps variable.

\section{Références}

[1] Z.-Q. Feng, 2D or 3D frictional contact algorithms and applications in a large deformation context, Comm. Numer. Methods Engrg. 11 (1995) 409-416.

[2] P. Joli, Z.-Q. Feng, Uzawa and Newton algorithms to solve frictional contact problems within the bi-potential framework, Int. J. Numer. Methods Engrg. 73 (2008) 317-330.

[3] Z.-Q. Feng, B. Magnain, J.-M. Cros, Solution of large deformation impact problems with friction between Blatz-Ko hyperelastic bodies, Int. J. Engrg. Sci. 44 (2006) 113-126.

[4] J.O. Hallquist, G.L. Goudreau, D.J. Benson, Sliding interface with contact-impact in Lagrangian computations, Comput. Methods Appl. Mech. Engrg. 51 (1985) 107-137.

[5] J.O. Hallquist, LS-DYNA Theory Manual, LSTC, Livermore Software Technology Corp., 2006.

[6] P. Wriggers, Computational Contact Mechanics, John Wiley \& Sons, 2002.

[7] J.O. Kim, B. Kwak, Dynamic analysis of two-dimensional frictional contact by linear complementarity problem formulation, Int. J. Solids Structures 33 (1996) 4605-4624. 